\title{
Program: XXVI Bárány Society Meeting
}

Thursday, August 19, 2010

\begin{tabular}{|c|c|c|c|c|}
\hline 08:30-09:00 & \multicolumn{4}{|c|}{ Opening Ceremony Háskólatorg Entry Hall (see separate Program) } \\
\hline 09:15-10:30 & $\begin{array}{c}\text { Room 102: Basic Science } \\
\text { Oral Session A1 } \\
\text { Advances \& Basic Science } \\
\text { Chairs: } \\
\text { Dan Merfeld } \\
\text { Paul Smith }\end{array}$ & $\begin{array}{c}\text { Room 103: Diagnosis } \\
\text { Oral Session B1 } \\
\text { Vestibular Loss } \\
\text { Chairs: } \\
\text { Miriam Welgampola } \\
\text { Brian Day }\end{array}$ & $\begin{array}{l}\text { Room 104: Disorders } \\
\text { Oral Session C1 } \\
\text { Specific Gravity } \\
\text { Chairs: } \\
\text { Leonardo Manzari } \\
\text { Giacinto Asprella }\end{array}$ & $\begin{array}{c}\text { Room 105: Treatment/Misc } \\
\text { Oral Session D1 } \\
\text { Drugs In Ear } \\
\text { Chairs: } \\
\text { Måns Magnusson } \\
\text { Takayuki Nakagawa }\end{array}$ \\
\hline 10:30-11:00 & \multicolumn{4}{|c|}{ Coffee } \\
\hline 11:00-12:30 & $\begin{array}{c}\text { Room 102: Basic Science } \\
\text { Oral Session A2 } \\
\text { VEMP } \\
\text { Chairs: } \\
\text { Ian Curthoys } \\
\text { Sally Rosengren }\end{array}$ & $\begin{array}{l}\text { Room 103: Diagnosis } \\
\text { Oral Session B2 } \\
\text { Head Impulse } \\
\text { Chairs: } \\
\text { Michael Halmagyi } \\
\text { Konrad Weber }\end{array}$ & $\begin{array}{c}\text { Room 104: Disorders } \\
\text { Oral Session C2 } \\
\text { Motion Sickness } \\
\text { Chairs: } \\
\text { Bernard Cohen } \\
\text { Philippe Perrin }\end{array}$ & $\begin{array}{c}\text { Room 105: Treatment/Misc } \\
\text { Oral Session D2 } \\
\text { Ageing \& Balance } \\
\text { Chairs: } \\
\text { F. Owen Black } \\
\text { Ilmari Pyykkö }\end{array}$ \\
\hline $12: 30-13: 30$ & \multicolumn{4}{|c|}{ Lunch } \\
\hline 13:30-14:00 & \multicolumn{4}{|c|}{ Poster Session 1} \\
\hline 14:00-15:45 & $\begin{array}{c}\text { Room 102: Basic Science } \\
\text { Oral Session A3 } \\
\text { VEMP } \\
\text { Chairs: } \\
\text { Ian Curthoys } \\
\text { Sally Rosengren }\end{array}$ & $\begin{array}{c}\text { Room 103: Diagnosis } \\
\text { Oral Session B3 } \\
\text { Mathematical Modelling } \\
\text { Chairs: } \\
\text { Rolf Johansson } \\
\text { Thomas Mergner }\end{array}$ & $\begin{array}{l}\text { Room 104: Disorders } \\
\text { Oral Session C3 } \\
\text { Neuro \& Neck } \\
\text { Chairs: } \\
\text { Thomas Lempert } \\
\text { Eva-Maj Malmström }\end{array}$ & $\begin{array}{c}\text { Room 105: Treatment/Misc } \\
\text { Oral Session D3 } \\
\text { Epidemiology } \\
\text { Chairs: } \\
\text { Howard Hoffman } \\
\text { Scott Eggers }\end{array}$ \\
\hline 15:45-16:00 & \multicolumn{4}{|c|}{ Coffee } \\
\hline $16: 00-17: 00$ & & $\begin{array}{l}\text { Room 103: Diagnosis } \\
\text { Oral Session B3 } \\
\text { (continued) } \\
\text { Mathematical Modelling }\end{array}$ & & \\
\hline 17:00-18:00 & & hy & Meeting & \\
\hline
\end{tabular}




\section{Program: XXVI Bárány Society Meeting}

Friday, August 20, 2010

\begin{tabular}{|c|c|c|c|c|}
\hline $08: 45-10: 30$ & $\begin{array}{c}\text { Room 103: Basic Science } \\
\text { Oral Session A4 } \\
\text { Postural Control } \\
\text { Chairs: } \\
\text { Richard Fitzpatrick } \\
\text { Juichi Ito }\end{array}$ & $\begin{array}{c}\text { Room 102: Diagnosis } \\
\text { Oral Session B4 } \\
\text { Eye \& Head Movements } \\
\text { Chairs: } \\
\text { Jelte Bos } \\
\text { Krister Brantberg }\end{array}$ & $\begin{array}{c}\text { Room 105: Disorders } \\
\text { Oral Session C4 } \\
\text { Vestibular Loss \& Little } \\
\text { More } \\
\text { Chairs: } \\
\text { Michael Karlberg } \\
\text { Barry Seemungal }\end{array}$ & $\begin{array}{c}\text { Room 104: } \\
\text { Treatment/Misc } \\
\text { Oral Session D4 } \\
\text { Vestibular Reflexes } \\
\text { Chairs: } \\
\text { Thomas Mergner } \\
\text { Pierre-Paul Vidal }\end{array}$ \\
\hline $10: 30-11: 00$ & \multicolumn{4}{|c|}{ Coffee } \\
\hline $11: 00-12: 30$ & $\begin{array}{c}\text { Room 103: Basic Science } \\
\text { Oral Session A5 } \\
\text { Vestibular-Eye Interaction } \\
\text { Chairs: } \\
\text { Swee Aw } \\
\text { Kathleen Cullen }\end{array}$ & $\begin{array}{c}\text { Room 102: Diagnosis } \\
\text { Oral Session B5 } \\
\text { Meniere's } \\
\text { Chairs: } \\
\text { Neil Shepard } \\
\text { Floris Wuyts }\end{array}$ & $\begin{array}{c}\text { Room 105: Disorders } \\
\text { Oral Session C5 } \\
\text { BPPV } \\
\text { Chairs: } \\
\text { Adolfo Bronstein } \\
\text { Miriam Weigampola }\end{array}$ & $\begin{array}{c}\text { Room 104: } \\
\text { Treatment/Misc } \\
\text { Oral Session D5 } \\
\text { Vestibular } \\
\text { Rehabilitation } \\
\text { Chairs: } \\
\text { Helen Cohen } \\
\text { Johan Holmberg }\end{array}$ \\
\hline $12: 30-13: 30$ & \multicolumn{4}{|c|}{ Lunch } \\
\hline $13: 30-14: 00$ & \multicolumn{4}{|c|}{ Poster Session 2} \\
\hline $14: 00-15: 30$ & $\begin{array}{c}\text { Room 103: Basic Science } \\
\text { Oral Session A6 } \\
\text { Inner Ear Morphology } \\
\text { Chairs: } \\
\text { Helge Rask Andersen } \\
\text { Timothy Hullar }\end{array}$ & $\begin{array}{c}\text { Room 102: Diagnosis } \\
\text { Oral Session B6 } \\
\text { ESCEBD \& The Young } \\
\text { Ones } \\
\text { Chairs: } \\
\text { Philippe Perrin } \\
\text { Herman Kingma }\end{array}$ & $\begin{array}{c}\text { Room 105: Disorders } \\
\text { Oral Session C6 } \\
\text { Meniere's \& More } \\
\text { Chairs: } \\
\text { Ilmari Pyykkö } \\
\text { Ian Curthoys }\end{array}$ & $\begin{array}{c}\text { Room 104: } \\
\text { Treatment/Misc } \\
\text { Oral Session D6 } \\
\text { Vestibular } \\
\text { Rehabilitation } \\
\text { Chairs: } \\
\text { Helen Cohen } \\
\text { Brian Day }\end{array}$ \\
\hline 15:30-16:00 & \multicolumn{4}{|c|}{ Coffee } \\
\hline 16:00-18:00 & $\begin{array}{c}\text { Room 102: Basic Science } \\
\text { Oral Session A7 } \\
\text { Classification } \\
\text { Chairs: } \\
\text { Herman Kingma } \\
\text { Alexander Bisdorff } \\
\end{array}$ & & & \\
\hline
\end{tabular}




\section{Program: XXVI Bárány Society Meeting}

Saturday, August 21, 2010

\begin{tabular}{|c|c|c|c|c|}
\hline $09: 30-10: 30$ & $\begin{array}{c}\text { Room 102: Basic Science } \\
\text { Oral Session A8 } \\
\text { Vestibular Physiology } \\
\text { Chairs: } \\
\text { Cynthya Darlington } \\
\text { Dan Merfeld }\end{array}$ & $\begin{array}{c}\text { Room 103: Diagnosis } \\
\text { Oral Session B7 } \\
\text { Differential Diagnosis } \\
\text { Chairs: } \\
\text { Nicolas Perez } \\
\text { Pierre Bertholon }\end{array}$ & $\begin{array}{c}\text { Room 104: Disorders } \\
\text { Oral Session C7 } \\
\text { Ageing \& Balance } \\
\text { Chair: } \\
\text { John Allum }\end{array}$ & $\begin{array}{c}\text { Room 105: Treatment/Misc } \\
\text { Oral Session D7 } \\
\text { Meniere's \& SSCD } \\
\text { Chairs: } \\
\text { Swee Aw } \\
\text { Martin Westhofen }\end{array}$ \\
\hline $10: 30-11: 00$ & \multicolumn{4}{|c|}{ Coffee } \\
\hline $11: 00-12: 30$ & $\begin{array}{c}\text { Room 102: Basic Science } \\
\text { Oral Session A8 } \\
\text { (continued) } \\
\text { Vestibular Physiology }\end{array}$ & $\begin{array}{l}\text { Room 103: Diagnosis } \\
\text { Oral Session B7 } \\
\quad \text { (continued) } \\
\text { Differential Diagnosis }\end{array}$ & $\begin{array}{l}\text { Room 104: Disorders } \\
\text { Oral Session C7 } \\
\text { (continued) } \\
\text { Ageing \& Balance }\end{array}$ & $\begin{array}{c}\text { Room 105: Treatment/Misc } \\
\text { Oral Session D7 } \\
\text { (continued) } \\
\text { Meniere's \& SSCD }\end{array}$ \\
\hline $12: 30-13: 30$ & \multicolumn{4}{|c|}{ Lunch/End of Congress } \\
\hline
\end{tabular}

